\title{
Vertebral Fractures of Unknown Origin: Role of Computed Tomography-Guided Biopsy
}

\author{
PAOLO SPINNATO, MD,${ }^{1}$ ALBERTO BAZZOCCHI, MD, PHD,${ }^{1}$ GIANCARLO FACCHINI, MD,${ }^{1}$ \\ GIACOMO FILONZI, MD, ${ }^{1}$ CRISTINA NANNI, MD ${ }^{2}$ ILARIA RAMBALDI, MD ${ }^{2}$ \\ EUGENIO RIMONDI, MD, ${ }^{1}$ STEFANO FANTI, MD, ${ }^{2}$ UGO ALBISINNI, MD ${ }^{1}$ \\ ${ }^{I}$ Diagnostic and Interventional Radiology, IRCCS Instituto Ortopedico Rizzoli, Bologna, Italy, ${ }^{2}$ Nuclear Medicine Unit, S. Orsola-Malpighi University Hospital, \\ Bologna, Italy
}

\begin{abstract}
Background: We performed a retrospective evaluation of histological and imaging results of patients submitted to computed tomography (CT)-guided biopsy for vertebral fractures (VFs) of unknown etiology to evaluate the pathological causes of fractures and also to observe the diagnostic results of imaging studies available.

Methods: We retrospectively reviewed all the CT-guided vertebral biopsies performed in our institution in the last 2 years, selecting patients with VF of unknown etiology. We reviewed clinical records, imaging studies, and histological examination results. We compared diagnostic performance of the 2 most sensitive imaging modalities for detection of malignancy on the collapsed vertebral body: magnetic resonance imaging (MRI) and positron emission tomography-CT (PET-CT). Anatomopathological results have been considered the gold standard to assess the diagnostic performance of imaging studies. Age stratification has been performed to understand the distribution of different anatomopathological diagnoses in age groups.

Results: Among 282 CT-guided vertebral biopsies, 36 (12.8\%) have been performed to diagnose the etiology of VF of unknown origin. In 26/32 (81.3\%), the vertebral biopsy was diagnostic: 8 osteopenia, 6 multiple myelomas, 4 osteomyelitis, 2 eosinophilic granuloma, 3 metastases, 1 mastocytosis, 1 Paget's disease, and 1 dysmielopoiesis. In 6 cases, the anatomopathological diagnosis was normal bone structure, most likely excluding malignancy. There were no statistically significance differences between MRI and PET-CT results $(P=1.0000)$.

Conclusions: Multiple myeloma and osteopenia represent the most frequent causes of this condition in adult patients, while eosinophilic granuloma and osteomyelitis in pediatric patients. Computed tomography-guided biopsy permits one to reach diagnosis in most of cases. Both PET and MRI could be insufficient to discriminate benign from malignant causes of fractures. Computed tomography-guided biopsy is needed when the etiology of fracture remains unclear.
\end{abstract}

Other \& Special Categories

Keywords: spinal fractures, image-guided biopsy, multidetector computed tomography, osteoporotic fractures, spontaneous fractures

\section{INTRODUCTION}

Nontraumatic vertebral fractures (VFs) are a relatively common finding, especially in elderly patients and in postmenopausal women. In the majority of cases, they are attributable to a condition of osteoporosis, although other diseases such as neoplasm and infections can also lead to VFs. ${ }^{1}$ In the pediatric population, nontraumatic VFs are rarer than in elderly patients, and the spectrum of diagnosis is significantly different, mainly concerning inflammatory diseases rather than metabolic or malignant. ${ }^{2}$

Nevertheless, in a small percentage of these fractures, the causes remain undetermined after clinical, laboratorial analyses, and also after the results of diagnostic imaging procedures, both in pediatric and adult patients. Differentiating between benign and malignant VFs has important therapeutic and prognostic implications. ${ }^{3}$

Conventional radiograph (XR) of the spine is the first diagnostic approach to these patients after clinical, laboratory, and anamnestic evaluation. Conventional radiograph shows some difficulties in distinguishing whether the fracture represents a consequence of osteoporosis, a metastatic lesion, or primary bone neoplasm. Computed tomography (CT) offers more information, helping in the evaluation of the degree of cortical bone destruction. ${ }^{4}$ Magnetic resonance imaging (MRI) is con- 
sidered the most helpful imaging tool to provide the basis for the distinction between neoplastic and acute osteoporotic fractures. Nevertheless, MRI can hardly lead to the correct diagnosis if strong bone edema is present. ${ }^{5}$ Positron emission tomographyCT (PET-CT) represents a very useful tool that can help to differentiate benign and malignant vertebral collapse, and it is able to evaluate the entire skeletal system and also extraskeletal regions in a single examination. However, PET-CT results could be positive in different pathological conditions, both in malignant and benign vertebral collapse. ${ }^{3}$

Computed tomography-guided vertebral biopsy (CTgVB) is known to be a safe and effective procedure to establish the correct diagnosis of spinal lesions and showed a higher success rate than fluoroscopic guided spinal biopsy. ${ }^{6,7}$

The aim of our study was to observe the diagnostic results of CTgVB in patients with VFs of uncertain etiology. More generally, our purpose was also to evaluate the diagnostic management of these patients, the role of diagnostic imaging tools available with particular regard on PET-CT studies, and also to analyze the causes of these fractures.

\section{MATERIALS AND METHODS}

We retrospectively reviewed all the 282 CTgVBs performed in our institution in the last 2 years, including in the study only patients submitted to $\mathrm{CTgVB}$ for the diagnosis of VFs of uncertain cause. The criteria of inclusion in the study were unclear radiologic features of VFs at previous examinations (XR, CT, and MRI) and/or clinical history at anamnesis that complicated diagnosis.

We reviewed clinical records, imaging studies, and histological examination results of patients with nontraumatic VFs of uncertain etiology submitted to $\mathrm{CTgVB}$. Vertebral fractures have been identified as loss in vertebral body height with visual semiquantitative assessment performed on XR and $\mathrm{CT}$ in consensus by 2 expert skeletal radiologists.

All biopsies have been performed by expert skeletal interventional radiologists in our institution. Anatomopathological result has been considered the gold standard to assess the diagnostic performance of all the imaging studies available. Additional immunohistochemical analyses on biopsy specimen have been performed in selected cases (especially in metastases and other neoplastic lesions).
Age stratification has been performed to understand the distribution of different anatomopathological diagnoses in different age groups: pediatric patients ( $0-17$ years), young adults (18-45 years), middle-aged adults (45-65 years), and elderly patients $(>65$ years).

The study was conducted following the Declaration of Helsinki with local institution review board approval.

\section{Technical Notes}

All biopsies have been performed with 8-, 11-, or 14-gauge biopsy needles, length: $10-15 \mathrm{~cm}$.

Computed tomography images of both diagnostic and biopsy procedures have been acquired with Brilliance CT 16 slice (Philips Medical Systems, Cleveland, $\mathrm{OH})$.

Fluorodeoxyglucose (FDG) PET-CT: Wholebody FDG PET-CT was carried out using standard procedures. Briefly, 3 to $5.7 \mathrm{MBq} / \mathrm{kg}$ of $18 \mathrm{~F}-\mathrm{FDG}$ were intravenously injected; 6 hours of fasting was required for all patients before injection. Antidiabetic therapy was discontinued during the day of the examination. The uptake time was 60 minutes for all patients. Images were acquired on a 2-dimensional (2D) tomographic mode (GE, Discovery LS; GE Healthcare Technologies, Milwaukee, WI) for 4 minutes/bed position or on a 3-dimensional (3D) tomographic mode (GE, Discovery STE; GE Healthcare Technologies) for 2 minutes/bed position. Lowdose CT $(120 \mathrm{kV}, 80 \mathrm{~mA})$ was performed, both for attenuation correction and as an atomical map.

Criteria to define PET-CT positivity included the following: either presence of focal areas of detectable increased tracer uptake within the spine (eg, more intense than background bone marrow uptake) excluding articular processes, with or without any underlying lesion identified by $\mathrm{CT}$, or a standardized uptake value maximum based on body weight accordingly to standard formula $\geq 2.5$ within osteolytic CT areas exceeding $1 \mathrm{~cm}$ in size or $>1.5$ within osteolytic $\mathrm{CT}$ areas ranging between 0.5 and $1 \mathrm{~cm}$ in size.

Magnetic resonance imaging studies have been performed in several medical centers with different equipment but all with 1.5 Tesla magnet with standard protocol.

\section{Statistical Methods}

Results are reported as frequencies or mean and standard deviation. Data were analyzed by $\mathrm{McNe}-$ 


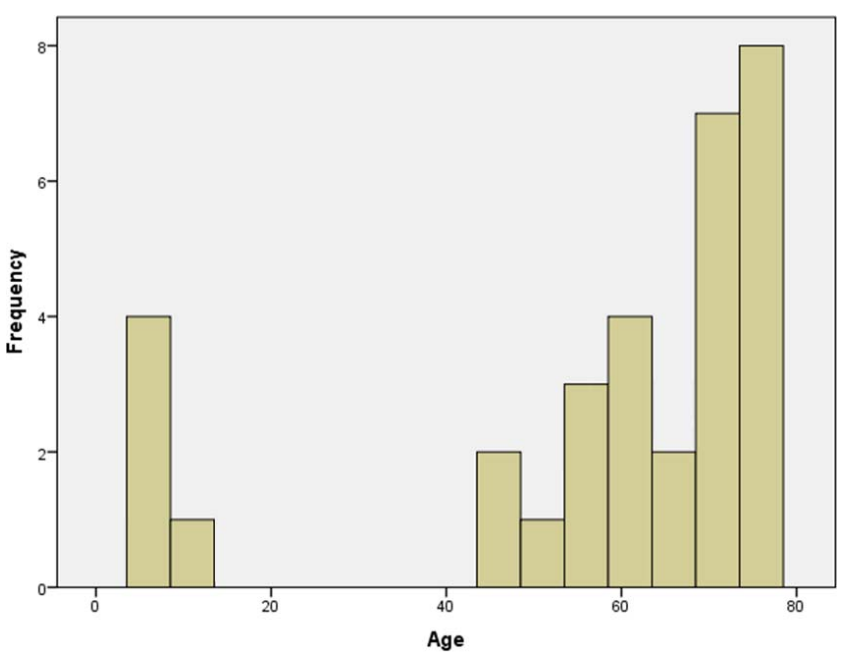

Figure 1. Distribution of patients' age.

mar's test, Fisher's test, $\chi^{2}$ test, normality test (Shapiro-Wilk test and Kolmogorov-Smirnov test). Two-tailed $P$ was considered significant for values less than .05 .

The SPSS (version 21; Armonk, New York) was used to perform the statistical tests mentioned above.

\section{RESULTS}

Among $282 \mathrm{CTgVBs}$ performed in our institution in the last 2 years, 135 have been carried out to diagnose vertebral expansive lesions clearly detected on radiological studies in patients without history of malignancy $(135 / 282,47.8 \%$; concomitant VFs in $28.9 \%$ of them), 71 to confirm spondylodiscitis also with microbiological analysis $(71 / 282,25.2 \%$; concomitant VFs in $36.6 \%$ of them), 40 to confirm a suspected vertebral metastasis or local recurrence in patients with history of malignancy (40/282, 14.2\%; with concomitant VFs in $45 \%$ of them), and only 36 $(36 / 282,12.8 \%)$ have been requested to differentiate the cause of VFs of unknown origin. Considering all the 282 CTgVBs, $119(119 / 282,42.2 \%)$ have been performed on VFs, and 36 of these have been carried out in VFs of unclear origin (36/119, $30.3 \%$ ). Thirty-six CTgVBs have been performed on 32 patients (mean age $57.1 \pm 23.3$ years, range 6-78 years; 13 males and 19 females), Figure 1 . The patients presented 1 (20 patients, $20 / 32,62.5 \%$ ) or more than 1 (12 patients, 12/32, 37.5\%) nontraumatic VFs. In 26 of 32 patients $(81 \%)$, pathological examination of the biopsy specimens was diagnostic (Figure 2): 8 bone thinning, osteopenia $(8 / 26,30.8 \%), 6$ multiple myeloma lesions $(6 /$

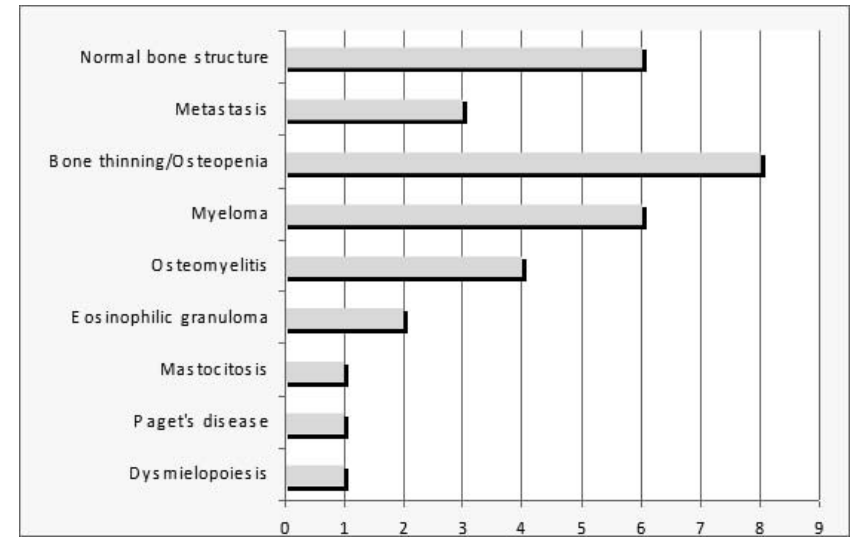

Figure 2. Anathomopathological results.

26, 23.1\%), 4 osteomyelitis $(4 / 26,15.4 \%), 2$ eosinophilic granuloma $(2 / 26,7.7 \%), 2$ lung cancer metastases $(2 / 26,7.7 \%), 1$ kidney cancer metastasis (1/26, 3.8\%), 1 mastocytosis $(1 / 26,3.8 \%), 1$ Paget's disease $(1 / 26,3.8 \%)$, and 1 dysmielopoiesis secondary to aspecific systemic disease $(1 / 26,3.8 \%)$. In the other 6 patients $(6 / 32,18.7 \%)$, the anatomopathological examinations showed normal trabecular and medullar bone structure. In 1 of them, the surgical biopsy confirmed normal bone structure. In 4 of these patients, the VFs were subsequently considered as nonneoplastic, reviewing clinical, laboratorial, and imaging studies together with the anatomopathological results. In 1 patient (male, 8 years old), $\mathrm{CTgVB}$, which was repeated twice (on $\mathrm{T} 8$ and T11 vertebra), excluded neoplasm or infections, but the cause remain unexplained. A PET-CT scan
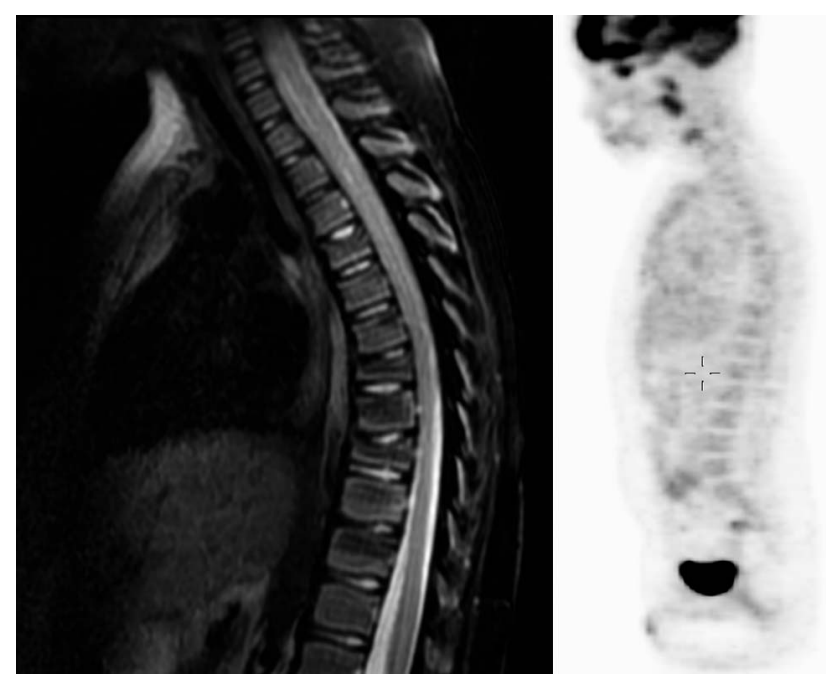

Figure 3. Magnetic resonance imaging study (sagittal fat suppression T2 weighted on the left) showed multiple vertebral fractures in an 8-year-old patient; PET-CT was negative (on the right). Biopsy has been performed on 2 different vertebral bodies without any diagnosis (normal bone structure). 


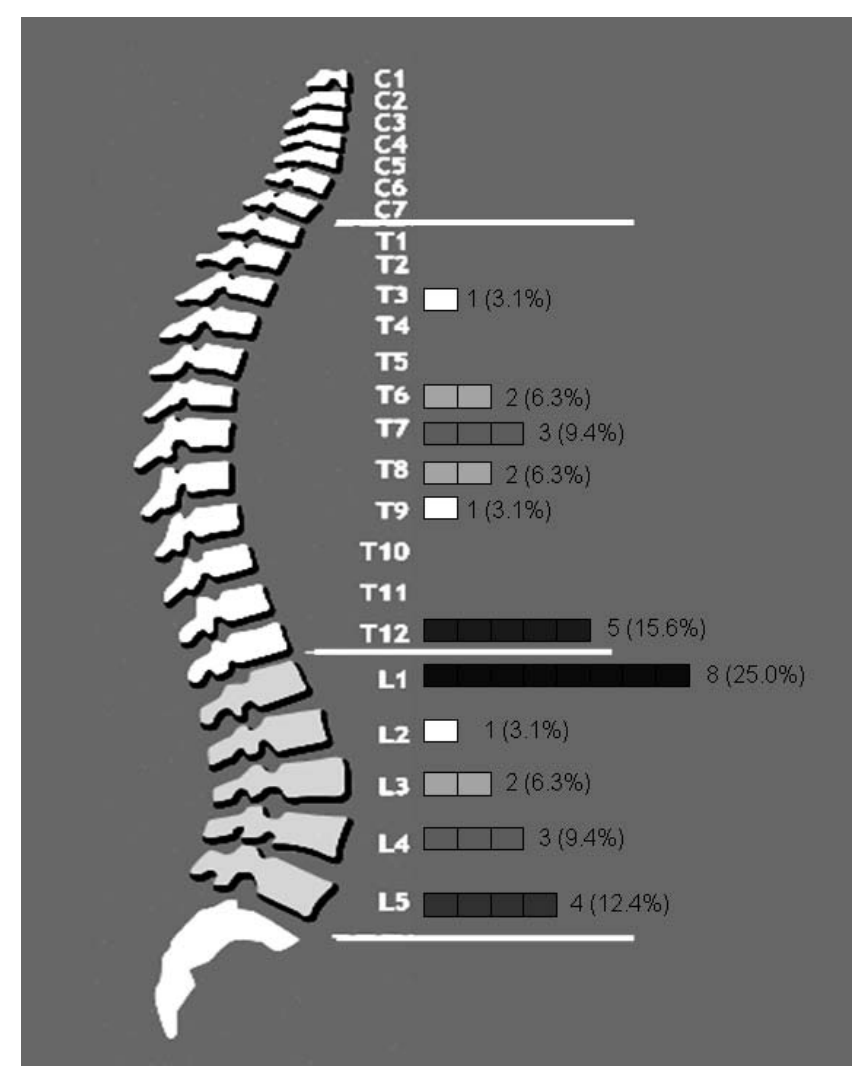

Figure 4. Computed tomography-guided vertebral biopsy level (not considering procedure's repetitions).

was negative, and MRI found no focal lesions, but bone edema in multiple vertebrae (Figure 3).

Twelve patients out of $32(37.5 \%)$ presented history of malignancy, and only in 2 of them $(2 / 12$, $16.7 \%$ ) metastases have been detected on the collapsed vertebrae. In the other 20 patients $(20 /$ $32,62.5 \%$ ) without history of malignancy, neoplasms have been diagnosed 7 times $(7 / 20,35.0 \% ; 1$ metastatic lesion and 6 multiple myeloma lesions).

In 4 out of 32 patients $(12.5 \%)$, physicians repeated biopsies, and in 1 of them, the diagnosis of multiple myeloma was obtained at the second histological evaluation. In the other 3 patients submitted to a second CTgVB. the first diagnosis was confirmed (osteomyelitis, eosinophilic granuloma, and normal bone structure). In the 2 patients with eosinophilc granuloma, this condition was clinically suspected, and extemporary anatomopathological evaluation was planned and performed together with a further corticosteroid injection on vertebral body using the same needle.

Our patients' ages were not normally distributed $(P$ $=.000$ using the Shapiro-Wilk test and $P=.003$ using the Kolmogorov-Smirnov test). In young adults, atraumatic VFs are rare, and there were none in our series between 11 and 44 years of age (Figure 1).

In patients presenting more than $1 \mathrm{VF}$, the frequency of malignancy seems to be lower than the frequency of malignancy in patients with a single $\mathrm{VF}$, even if there is no statistically significant correlation in our limited group of patients $(P=$ .077). And in the same way, the higher the number of VFs, the higher the probability of a benign diagnosis $(P=.088)$.

Vertebral biopsies have been performed on different vertebral bodies as shown in Figure 4, not considering procedure repetition.

No complications have been observed after biopsy procedures.

Twenty-four patients submitted to CTgVBs also underwent a standard FDG PET-CT whole body evaluation. Regarding functional imaging, FDG PET-CT turned out positive in $15 / 24$ patients. Twelve scans were true positive (malignancy or inflammation), 7 true negative, 2 false negative, and 3 false positive, leading to an overall sensitivity of $86 \%$, specificity of $70 \%$, and accuracy of $80 \%$.

In particular, FDG PET-CT was falsely negative in a mature plasmacytoma and eosinophilic granuloma, while it was falsely positive in 3 patients with normal bone structure.

Magnetic resonance imaging suspected neoplastic fracture in 4 patients with negative pathological results ( 3 bone thinning and 1 normal bone structure). Moreover, MRI did not detect any lesion in 4 patients with malignant VFs ( 3 myeloma lesions and 1 metastatic lesion), and 3 of them (75\%) presented bone edema in the collapsed vertebra.

Considering patients submitted to both MRI and PET-CT examinations, there were no statistically significant differences between the 2 techniques' results in detecting malignancy on the collapsed vertebrae $(P=1.0000)$.

Considering separately pediatric patients enrolled in the study, we had 5 patients $(5 / 32,15.6 \%)$ who underwent $\mathrm{CTgVBs}$ for $\mathrm{VFs}$ of unknown origin. None of them presented history of malignancy. Anatomopathological results showed 2 osteomyelitis $(2 / 5,40 \%), 2$ eosinophilic granuloma $(40 \%)$, and 1 normal bone structure with biopsy repeated with the same result excluding malignancy $(10 \%)$.

No young adult patients (considering 18-40 years range) have been enrolled in the study (no patients found between 11 and 44 years old). 


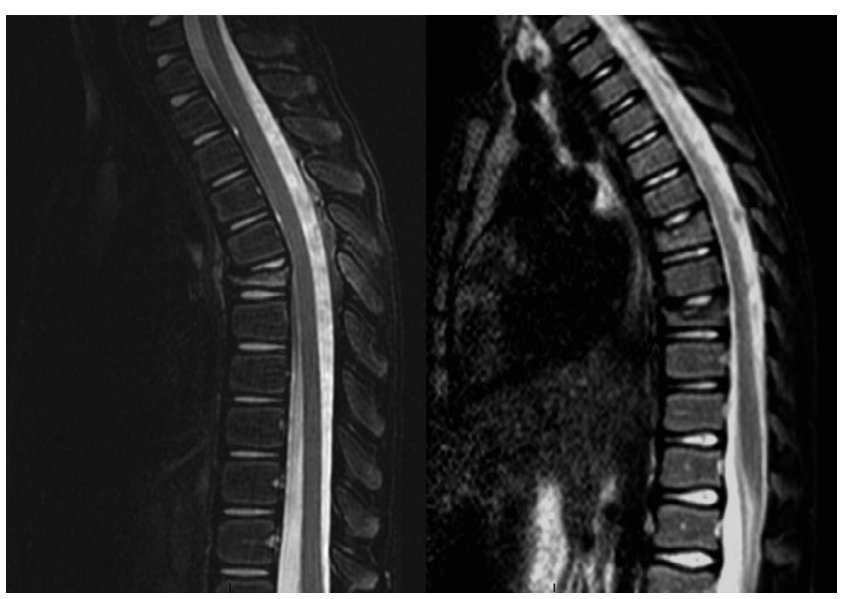

Figure 5. Magnetic resonance imaging studies (sagittal fat suppression T2 weighted) showed fracture of T6 in a 6-year-old patient (on the left) and fracture of T9 and T11 in an 8-year-old patient (on the right) with similar radiologic patterns but different anatomopathological results: osteomyelitis (on the left) and eosinophilic granuloma (on the right).

Considering separately middle-aged adults (45-65 years old) enrolled in the study, we had 12 patients $(12 / 32,37.5 \%)$. Three of them presented history of malignancy, and in 1 , metastatic involvement was found $(1 / 3,33.3 \%)$. None of them presented a known condition of osteoporosis. Anatomopathological results showed 4 myeloma lesions (4/12, $33.3 \%), 3$ osteopenia/bone thinning $(25.0 \%), 1$ Paget's disease $(8.3 \%), 1$ mastocytosis $(8.3 \%), 1$ dysmielopoiesis secondary to aspecific systemic disease $(8.3 \%), 1$ normal bone structure $(8.3 \%)$, and 1 metastases $(8.3 \%)$.

Considering separately elderly patients $(>65$ years old) enrolled in the study, we found 15 patients $(15 / 32,46.9 \%)$ submitted to CTgVB for VFs of unknown etiology. Six of them presented history of malignancy, and in 2, metastatic involvement was found $(2 / 6,33.3 \%)$. Anatomopathological results showed 5 osteopenia/bone thinning $(5 / 15$, $33.3 \%), 4$ normal bone structure $(26.7 \%), 2$ osteomyelitis/spondylodiscitis (13.3\%), 2 myeloma lesions $(13.3 \%)$, and 2 metastases $(13.3 \%)$.

\section{DISCUSSION}

Confirming the diagnosis of atraumatic VFs in seniors with a past history of malignancy and osteoporosis can present a clinical challenge, as reported by other previous studies. ${ }^{3,8}$ Moreover, it has been already reported in the literature by several studies that, in presumed osteoporotic VFs after histological analysis, an unexpected diagnosis of malignancy can occur in about $4-5 \%$ of patients. ${ }^{9,10}$ In elderly patients, the goal is to discriminate

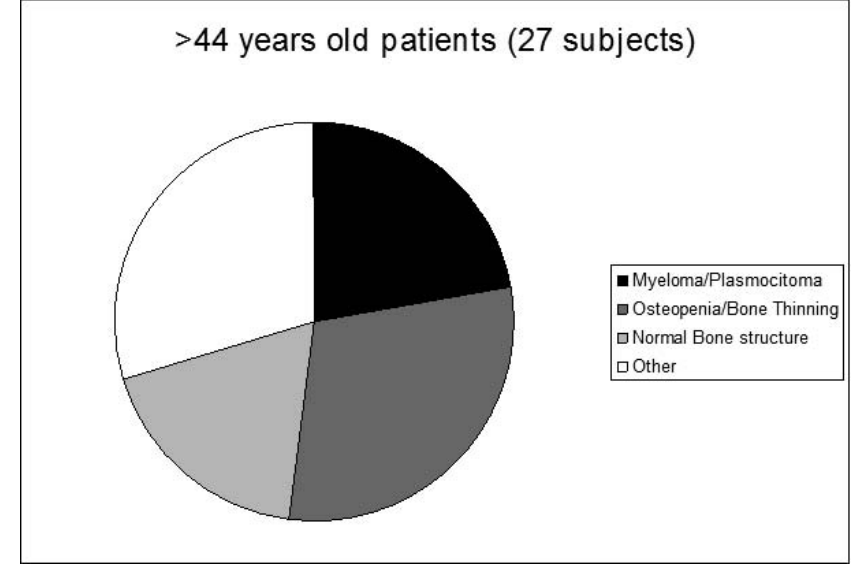

Figure 6. Anatomopathological diagnosis in patients 44 years old or older (range 44-78 years).

malignant VFs from osteoporotic fractures, and CTgVB is the appropriate tool when doubtful imaging findings occur, especially if a history of malignancy is present. We also found 2 spondylodiscitis in elderly patients included in the study that were not suspected after clinical and radiological evaluations.

In our experience, it could be difficult to diagnose the correct cause of VFs also in pediatric patients (Figure 5). Even if the spectrum of pathologies is limited in this population, the correct diagnosis is essential to carry out the appropriate therapeutic strategies.

Computed tomography-guided vertebral biopsy seems to be appropriate and safe for patients with nontraumatic VFs of unknown etiology, showing high diagnostic performance. Furthermore, in many patients, this procedure could be the only available diagnostic tool, especially when clinical, radiological, and laboratorial findings are not able to identify the cause of VFs.

\section{$<11$ years old patients (5 subjects)}

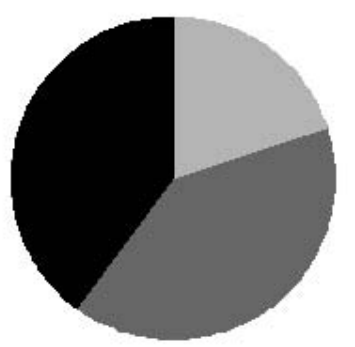

Normal bone structure

- 5 pondylodis citis/O s te omyelitis

- E os inophilic granuloma

Figure 7. Anatomopathological diagnosis in patients 10 years old or younger (range 6-10 years). 
According to our results, osteoporosis and multiple myeloma lesions seem to be the most common causes of idiopathic nontraumatic VFs, especially in patients older than 45 years (Figure 6). Considering the pediatric patients included in our study, represented by 10 -year old patients or younger, osteomyelitis and eosinophilic granuloma are the most common cause of nontraumatic VFs (Figure 7).

Regarding the different anatomopatholgical results found in various age stages, we see that malignant diagnoses are more frequent in the middle-aged adult population $(41.7 \%)$ than in the elderly $(26.7 \%)$ and children $(0 \%)$. As expected, anatomopathological signs of bone thinning/osteopenia are more frequent in elderly patients $(33.3 \%)$ than in middle-aged adults $(25.0 \%)$ and children $(0 \%)$. These results confirm that, in young populations, osteoporotic fractures are rare, and the occurrence of nontraumatic VFs strongly suggests other pathologic conditions (neoplasm, infections/ inflammations). The radiological evidence of reduction in the vertebral body's height in young patients with normal radiological appearance of bone structure (without signs of bone loss) must be considered suspect, and further analyses are recommended. Computed tomography-guided vertebral biopsy is appropriate and useful in these cases, especially if suspicious clinical findings such as back pain resistant to medical therapy, presence of inflammatory and/or tumoral markers, and weight loss are present.

Only in 3 patients $(3 / 32,9.3 \%)$, the cause of undetermined VFs was represented by a metastatic bone involvement, perhaps due to the good performance of diagnostic examinations (especially MRI and $\mathrm{CT}$ ) in the detection of focal lesions in fractured vertebrae. $^{5}$

However, in a small percentage of patients, the etiology of VFs remains unknown, but in these cases after CTgVB with nondiagnostic results, malignancies are most likely excluded, and imaging and clinical follow up is sufficient.

Regarding functional imaging, FDG PET-CT was proved to provide good results in characterizing the cause of the fracture (inflammation/malignancy versus osteoporotic). Of course, we had some false positive results probably related to a recent fracture still showing inflammatory aspects and a false negative related to well-differentiated malignancies (that are usually low metabolic) or chronic inflammation. Of course, FDG PET-CT cannot differen-

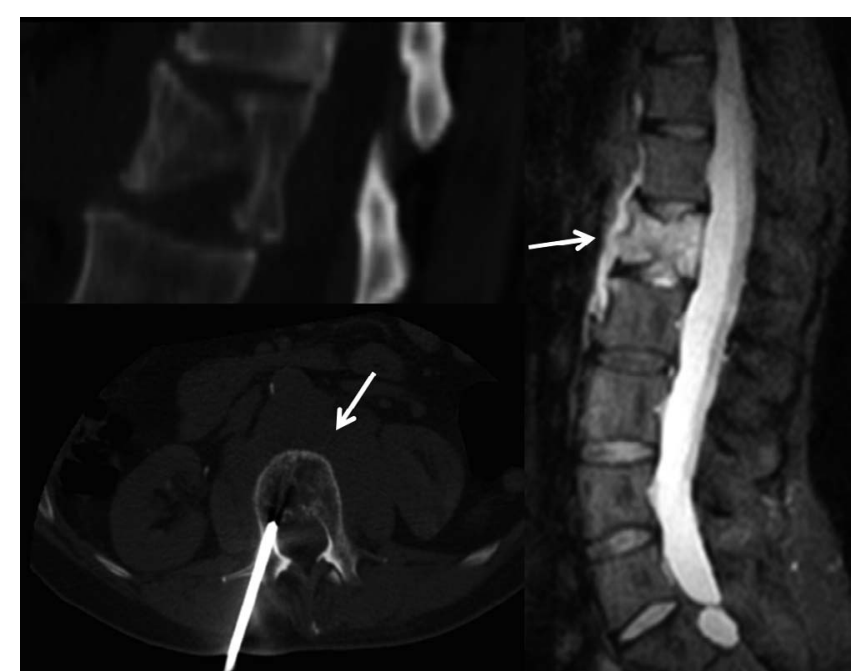

Figure 8. Magnetic resonance imaging showed recent L1 fracture with strong bone edema (short tau inversion recovery sagittal sequence; on the right) and fluid in paravertebral space (arrows), without focal lesions detectable. Computed tomography confirmed the fracture (upper left). Computed tomography-guided vertebral biopsy, performed on L1 (down left), revealed multiple myeloma.

tiate benign (inflammatory) versus malignant vertebral collapse due to the aspecific uptake mechanism of FDG, which is enhanced both in inflammation and cancer. Moreover, false negative PET-CT results on multiple myeloma lesions are expected, as occurred to 1 patient of our study, and as showed by other previous researches at diagnosis of the disease especially in diffuse or micronodular spine involvement. ${ }^{11,12}$

Magnetic resonance imaging could be insufficient, especially if strong edema is present on the collapsed vertebra (Figure 8). Recent studies showed the good diagnostic potential of 3 Tesla MRI machines in differentiating benign from malignant VFs, particularly using diffusion-weighted imaging. ${ }^{13}$

The limits of this study are represented by the retrospective nature of the research, the few number of patients, and the MRI studies performed on different types of equipment.

Computed tomography-guided vertebral biopsy in most cases leads to the diagnosis of the disease and can help in choosing in a short time the appropriate treatment strategies for these patients. However, $\mathrm{CTgVB}$ is an invasive procedure, and in our opinion, it must only be performed only after accurate clinical, laboratory, and anamnestic evaluation of these patients and when imaging diagnostic tools (XR, CT, MRI, and possibly PET) do not certainly individuate the cause of nontraumatic VFs. In selected cases, CTgVB could be requested 
by oncologists also in most likely metastatic VFs if it is necessary to define the immunohistochemical features of lesions to assess the correct therapeutic strategies.

\section{REFERENCES}

1. Alexandru D, So W. Evaluation and management of vertebral compression fractures. Perm J. 2012;16(4):46-51.

2. Hirji H, Saifuddin A. Paediatric acquired pathological vertebral collapse. Skeletal Radiol. 2014;43(4):423-436.

3. Bredella MA, Essary B, Torriani M, Ouelette HA, Palmer WE. Use of FDG-PET in differentiating benign from malignant compression fractures. Skeletal Radiol. 2008;37(5):405-413.

4. Cicala D, Briganti F, Casale L, et al. Atraumatic vertebral compression fractures: differential diagnosis between benign osteoporotic and malignant fractures by MRI. Musculoskelet Surg. 2013;97(Suppl 2):S169-S179.

5. Baur-Melnyk A. Malignant versus benign vertebral collapse: are new imaging techniques useful? Cancer Imaging. 2009;9 Spec No A:S49-S51.

6. Cherdchukiatsakul S. The results of comparisons between CT-guided and fluoroscopic-guided spinal biopsy. J Med Assoc Thai. 2013;96 Suppl 3:S59-S63.

7. Heyer CM, Al-Hadari A, Mueller KM, Stachon A, Nicolas V. Effectiveness of CT-guided percutaneous biopsies of the spine: an analysis of 202 examinations. Acad Radiol. 2008;15(7):901-911.

8. Fayad LM, Kamel IR, Kawamoto S, Bluemke DA, Frassica FJ, Fishman EK. Distinguishing stress fractures from pathologic fractures: a multimodality approach. Skeletal Radiol. 2005;34(5):245-259.

9. Muijs SP, Akkermans PA, van Erkel AR, Dijkstra SD. The value of routinely performing a bone biopsy during percutaneous vertebroplasty in treatment of osteoporotic vertebral compression fractures. Spine (Phila Pa 1976). 2009;34(22):2395-2399.

10. Mukherjee S, Thakur B, Bhagawati D, et al. Utility of routine biopsy at vertebroplasty in the management of vertebral compression fractures: a tertiary center experience. J Neurosurg Spine. 2014;21(5):687-697.

11. Spinnato P, Bazzocchi A, Brioli A, et al. Contrast enhanced MRI and ${ }^{18}$ F-FDG PET-CT in the assessment of multiple myeloma: a comparison of results in different phases of the disease. Eur J Radiol. 2012;81(12):4013-4018.

12. Zamagni E, Nanni C, Patriarca F, et al. A prospective comparison of $18 \mathrm{~F}$-fluorodeoxyglucose positron emission tomography-computed tomography, magnetic resonance imaging and whole-body planar radiographs in the assessment of bone disease in newly diagnosed multiple myeloma. Haematologica. 2007;92(1):50-55.

13. Sung JK, Jee WH, Jung JY, et al. Differentiation of acute osteoporotic and malignant compression fractures of the spine: use of additive qualitative and quantitative axial diffusion-weighted MR imaging to conventional MR imaging at 3.0 T. Radiology. 2014;271(12):488-498.

Disclosures and COl: Author contributions: Spinnato $\mathrm{P}$ designed and performed the research and wrote the paper; Bazzocchi A helped in designing the research and supervised the manuscript; Facchini G and Filonzi G collected data and supervised the manuscript; Rambaldi I and Nanni C helped in designing the research and contributed to the analysis and data collection; Rimondi E collected data and supervised manuscript; Fanti S and Albisinni $\mathrm{U}$ supervised and approved the manuscript.

This research was conducted following the Declaration of Helsinki because of the retrospective and observational nature of the study.

Informed consent statement: Patients were not required to give informed consent to the study because the analysis used anonymous clinical data that were obtained after each patient agreed to treatment by written consent.

We have no financial relationships to disclose. Data sharing statement: No additional data are available.

Corresponding Author: Paolo Spinnato, MD, Diagnostic and Interventional Radiology, "Rizzoli" Orthopaedic Institute, Bologna, Italy Via GC Pupilli n.1, 40136, Bologna, Italy. Phone: +39 051-6366836, +39 051-6366021; Fax: +39 0516366280; Email: paolo.spinnato1982@gmail.com, paolo.spinnato@ior.it.

Published 21 December 2018

This manuscript is generously published free of charge by ISASS, the International Society for the Advancement of Spine Surgery. Copyright (C) 2018 ISASS. To see more or order reprints or permissions, see http://ijssurgery.com. 